\title{
Philosophiques
}

\section{Le " féminisme de la frontière " entre djihad érotique et politique du care}

\section{AGNÈS BERTHELOT-RAFFARD}

Volume 44, numéro 1, printemps 2017

URI : https://id.erudit.org/iderudit/1040331ar

DOI : https://doi.org/10.7202/1040331ar

Aller au sommaire du numéro

Éditeur(s)

Société de philosophie du Québec

ISSN

0316-2923 (imprimé)

1492-1391 (numérique)

Découvrir la revue

Citer ce document

Berthelot-Raffard, A. (2017). Le « féminisme de la frontière » entre djihad érotique et politique du care. Philosophiques, 44(1), 109-115.

https://doi.org/10.7202/1040331ar d'utilisation que vous pouvez consulter en ligne.

https://apropos.erudit.org/fr/usagers/politique-dutilisation/ 


\title{
Le «féminisme de la frontière» entre djihad érotique et politique du care
}

\author{
AGNĖS BERTHELOT-RAFFARD \\ Professeure-adjointe \\ Institut d'études féministes et de genre \\ Université d'Ottawa \\ abraffard@gmail.com
}

Empruntant à différents champs de la philosophie politique et des études féministes, l'ouvrage de Soumaya Mestiri, Décoloniser le féminisme. Une approche transculturelle (2016) examine les conditions d'un "féminisme de la frontière». Affirmant la reconnaissance des différences fondamentales entre les femmes, l'autrice récuse un féminisme libéral occidental qui, tout en étant construit sur les rejets du caractère andocentrique des valeurs de liberté et d'égalité, reproduit l'hégémonie qu'il prétend contester. Construit sur la continuité d'une colonialité du pouvoir, il occulte l'apport des philosophies du Moyen-Âge et de la Renaissance, et n'inclut pas les luttes anticoloniales des féministes «indigènes» et chicanas dans la construction du féminisme contemporain. Contre de tels biais, Mestiri propose sa révision afin de donner une voix à celles que l'on pense différentes. Le «féminisme de la frontière" serait plus à même de provoquer la rencontre tant attendue entre l' "Occident" et l' "Orient ", sans toutefois enclaver ni provincialiser. Il ne nierait pas les limites et les fractures entre la périphérie et le centre, la province et la métropole. Il s'agirait, en ce sens, d'un féminisme produit par des théoriciennes et militantes conscientes des fractures et des rapports douloureux entre la marge et le centre. Plus que tout autre, il se préoccuperait de déconstruire la matrice du pouvoir. Davantage que le féminisme libéral, il serait en mesure de donner voix au chapitre à celles qui sont à la marge, sans chercher ni à les insérer au centre ni à les ramener de force à la périphérie. Ouvert à l'altérité qu'elles proposent par leurs points de vue, il leur permettrait enfin d'assumer leur héritage en rendant possible l'entremêlement de leurs voix avec celles émanant du canon dominant. Le «féminisme de la frontière » briserait ainsi la posture allant du savoir au pouvoir en proposant un déplacement épistémologique. Ce féminisme véritablement décolonial récuserait en particulier les injustices épistémiques qui participent à la perpétuation d'un pouvoir hégémonique. Il ne pourrait être obtenu qu'au prix de cette rupture. En effet, la colonialité du genre empêche la réciprocité entre les femmes et nuit à leur fédération. Elle contribue à annihiler le point de vue de celles qui, par leurs particularismes, ne peuvent prétendre à l'universalité du "sujet féminin ». Elle est donc l'expression d'une colonialité du pouvoir qui est aussi profondément épistémique.

Après un retour sur la démarche de Mestiri, deux principales critiques seront formulées sur: I) les modalités et les résultats de la décolonisation de 
Shéhérazade; et 2) les soubassements de la notion de "care au service de l'empowerment».

\section{Déconstruire la «colonialité du genre»}

Pour l'autrice, Décoloniser le féminisme relève d'une triple démarche. L'approche s'inscrit d'abord dans le constat des limites du féminisme majoritaire en raison de la colonialité du genre qui est une imposition plutôt qu'une émancipation. Ensuite, elle vise à déconstruire les stéréotypes sur les femmes musulmanes. Enfin, elle examine de façon métacognitive la décolonisation d'objets/sujets qui semblent déjà passés au crible de cette approche. L'ouvrage cherche notamment à repenser le récit colonial dans lequel la femme arabe est fantasmée, déshumanisée, hypersexualisée, asservie au mâle "barbare» puis nécessairement libérée et ré-asservie par un prétendu "sauveur blanc». Il questionne également la notion d'empowerment des femmes de la périphérie. Tout droit sorties des instances mettant en œuvre les plans d'ajustements structurels dans les zones les plus pauvres du globe, les composantes de l'empowerment relèvent selon Mestiri d'une colonialité $d u$ pouvoir qui assoit la fragmentation des femmes selon leur classe sociale, leur localité dans l'espace-monde, leurs capacités réflexives, leur "race» et leur religion. Pour montrer les apories de l'empowerment, l'autrice s'intéresse au care comme éthique et comme pratique qui prétend supplanter ce concept. La proposition de fonder un "féminisme de la frontière" s'avère un objectif ambitieux, et Mestiri soutient cette démarche décoloniale en arrimant son approche transculturelle à une critique en règle de la philosophie libérale contemporaine. Cette posture est inédite dans le champ des études féministes postcoloniales et transnationales.

Parmi la profusion d'ouvrages parus récemment sur le féminisme décolonial, celui de Mestiri s'avère original pour deux raisons majeures: son refus d'endosser une fausse neutralité épistémique dans un domaine où cette posture intellectuelle est la règle, et sa démarche de déconstruction de l'Orient et de ses sujets. Sur le premier point, l'ouvrage s'inscrit dans la discussion sur les limites de la discipline philosophique et son manque de neutralité épistémique sur les sujets qui concernent les «dominé.e.s» et/ou qui sont produits par eux. Visant une décolonisation épistémique, Mestiri invite le lecteur à se questionner sur les pratiques d'intégration des savoirs de ceux que la philosophie hégémonique tend à considérer comme "subalternes". Dans cette optique, l'autrice énonce qu'elle n'est pas neutre quant aux sujets qu'elle traite. Elle refuse d'endosser la fausse neutralité que réclame l'épistémologie positiviste et dans laquelle s'inscrit pleinement le raisonnement philosophique. Bien que cette théorie féministe ne soit pas mentionnée, la démarche de Mestiri n'est pas sans rappeler celle du Black Feminism. En effet, dès le prologue, l'autrice fait valoir la nécessité de tenir un "point de vue situé». Cependant, cette théorie féministe restera la grande absente de 
ce livre - ce qu'un lecteur averti pourrait déplorer. Cette exclusion est, de prime abord, étonnante pour deux raisons.

Premièrement, parce que l'autrice fait mention des théories féministes produites par les femmes africaines, les chicanas et les indiennes, et qu'elle aurait pu inclure les féministes africaines-américaines puisqu'elles prônent une alliance avec ces femmes du tiers-monde. Ensuite, l'étonnement provient du sujet qui traverse ce livre: la théorisation du pouvoir pour décoloniser les savoirs étant au cœur même des réflexions des féministes africaines-américaines et ce, depuis l'origine de ce mouvement. Cet oubli théorique semble toutefois témoigner d'un positionnement important. En effet, Mestiri s'intéresse aux féminismes pour les femmes que le féminisme occidental considère comme des "subalternes" alors que ces dernières se trouvent placées dans un contexte de production des savoirs et des pratiques militantes où elles sont majoritaires. En effet, l'ouvrage n'aborde pas les femmes racisées et musulmanes qui vivent dans des pays majoritairement blancs et judéo-chrétiens, bien qu'il s'avère éclairant pour comprendre leur positionnement singulier.

Le second point est d'ordre méthodologique. La démarche de Mestiri s'inscrit dans une posture épistémologique empruntant au témoignage et à la métaphore, ce qui contribue à coordonner la voix des femmes de la frontière avec sa voix. Cette posture apparaît d'emblée originale, car elle rompt partiellement avec la méthodologie traditionnelle et non empirique de la philosophie sociale occidentale, trop coincée entre une prétention à la normativité et un besoin d'ancrer ses domaines d'application dans une démarche plutôt casuistique. Ensuite, l'apport de la métaphore, si singulier soit-il, est symbolique. En effet, il fait écho à une vision fantasmée de l' "Orient» dont les savoirs sont réputés se transmettre par l'usage des proverbes et des récits. Mestiri utilise cette démarche, non pour se conformer à un possible orientalisme de son lecteur - posture on ne peut plus probable dans la mesure où elle s'adresse en premier lieu à un public plus familier avec les théories de la justice libérale contemporaine qu'avec la philosophie arabe médiévale. Paradoxalement, l'usage de la métaphore est une méthode de rupture avec un orientalisme qui érige les représentations stéréotypées en vérité. Bien que cette déconstruction paraisse nécessaire surtout en ces temps où l'orientalisme assoit, ici et ailleurs, une islamophobie fondée sur une grande ignorance des us et coutumes de ce qui est vu comme un Ailleurs, ce procédé de retour au mythe pour le déconstruire est questionnable. Il est particulièrement contre-productif s'agissant des efforts mis en œuvre pour briser l'image des femmes musulmanes qui nous est proposé dans le chapitre II.

\section{Quand faire l'amour, c'est faire le djihâd}

"Décoloniser Shéhérazade en donnant à voir sa supériorité, c'est aussi incarner le fantasme colonial jusqu'à lui tordre le cou» (p. 55). Aussi nous est-il proposé de procéder à une double déconstruction. Tout d'abord, nous 
sommes invités à rompre avec l'image de la femme musulmane telle qu'elle est véhiculée par les médias et formalisée par les pouvoirs publics occidentaux (archétype normatif $\mathrm{n}^{\circ} \mathrm{I}$ ). Ensuite, une rupture avec une vision sexualisée de la femme orientale propose de déconstruire la vision coloniale d'une femme hyper-sexualisée, laquelle justifie l'appropriation de celle-ci par l'homme blanc et son confinement dans un harem (archétype normatif $\left.n^{\circ} 2\right)$.

Représentée d'une manière stéréotypée, la femme arabe, pourtant loin d'être soumise, est cloisonnée dans la sphère privée, et sans droits. Elle est au contraire promotrice de sa sexualité. Elle ne se situe donc dans aucun des deux archétypes. En effet, comme les hommes, elle a le droit d'avoir du plaisir. Le plaisir participe d'une sacralisation de l'union dont l'homme et la femme bénéficient de façon égale. La liberté sexuelle est une des bases de l'équilibre du couple. Le sexe ne souille pas les amants. Le rapport sexuel est, au contraire, l'incarnation du djihâd. C'est un acte de piété comme un autre. Le lien qui unit, dans l'Islam, la religion et le sexe est entériné par un contrat qui accorde aux deux partenaires une propriété sexuelle égale. Parmi ces droits et ces devoirs figure la jouissance partagée et le consentement mutuel. Par ailleurs, bien que l'homme soit doté d'un pouvoir sur ses capacités viriles, la femme l'enrichit de son savoir supérieur en la matière. Ainsi, nous sommes très loin d'une vision étriquée et unilatérale des relations conjugales dans les couples musulmans. Ici, la sexualité féminine soutient la mutualité dont il sera davantage question dans le chapitre III. Le chapitre II rappelle par endroits certains passages du Mesnevi de Djalâl ad-Dîn Rûmî, dans lequel l'amoureux est la figure bénie d'Allah. Il fait également écho au Cantique des cantiques. Comme dans le monde judéo-chrétien, l'amour est, dans l'Islam, une ode à Dieu et rapproche de cette figure sacrée.

Si la seconde image de Shéhérazade déconstruit un mythe, la première procède au mouvement inverse, donnant le sentiment que Mestiri n'a finalement pas réussi à «tordre le cou » au fantasme. Celui-ci est incarné à l'excès. Mestiri a simplement incité ses lecteurs à le regarder dans le miroir pour soutenir la mise en abîme du mythe. Contemplant une Shéhérazade insoumise, experte et actrice d'un djihad érotique, le lecteur demeure dans une posture coloniale. Certes, nourri de la restitution de sources issues de la philosophie et de la théologie arabe médiévale, notamment Ibn Suleiman et Tifâchi, il semble plus averti. Conscient qu'il faut rompre avec une image occidentale de la femme musulmane soumise jusque dans sa sexualité, il comprend que, s'il y a soumission, elle ne peut être qu'un jeu ou une posture feinte. Il sait désormais qu'elle soutient un sens plus profond et plus symbolique que l'on ne saurait saisir en l'extrapolant de l'extérieur. Mais avait-on vraiment besoin de regarder par le trou de la serrure pour mieux comprendre ce qui se passe dans l'alcôve?

Bien que l'on comprenne que Mestiri se soit attaquée à une vision faussée du couple musulman, qu'elle ait voulu récuser une dichotomie 
masculin/féminin et restaurer sa mutualité, elle a participé à un double mouvement. D'abord, en proposant des sources orientales et en faisant référence au monde arabe et à sa culture, elle a reconduit un universalisme sur cette région du monde, encore mal délimitée dans certains esprits. Par ailleurs, bien que les exemples historiques tendent à montrer la liberté sexuelle des femmes musulmanes et à récuser une image de soumission à des hommes empêtrés dans une culture "barbarisante", elle nous parle de la situation actuelle et, dès lors, nous ne comprenons pas bien pourquoi, dans le chapitre IV, elle reproche aux féministes tunisiennes actuelles de se figer dans les revendications de la seconde vague du féminisme français sur l'égalité et la liberté sexuelle. Si la religion musulmane incite, dès son origine, à la liberté sexuelle des femmes, pourquoi devraient-elles encore la revendiquer?

Par ailleurs, en cherchant à déconstruire ces deux archétypes, Mestiri participe, paradoxalement, à la construction d'une autre image de la femme arabe. En cherchant à la restituer, elle participe à un processus obligé vécu par toutes les femmes dites «de couleur» lorsqu'elles adoptent une posture visant à rompre avec leur hypersexualité présumée. Se réapproprier sa sexualité et lutter contre les archétypes formulés à son propos conduit à un cercle vicieux que le raisonnement de Mestiri reproduit. À plusieurs reprises, elle affirme que la femme arabe est celle qui est experte de sa sexualité. Son savoir est son pouvoir de dire non à une relation non consentie, de provoquer le désir chez l'autre. C'est aussi un savoir détenu sur son propre corps, ses désirs, ses besoins et ses limites. Mais l'image de la femme arabe et la réappropriation de sa sexualité revisitée par Mestiri nous plongent dans une aporie discursive et épistémique. Comme les icônes de la pop culture américaine quand elles jouent avec leur sexualité pour en prouver l'auto-contrôle, la réappropriation de la sexualité suppose une nouvelle sexualisation qui ne casse pas les codes de l'hyper-sexualisation. Les attentes de ces icônes de la pop-culture sont seulement détournées par le jeu de déconstruction. Le problème est que ce jeu est aporétique, car il ne change pas l'actoricité des agents. Si la femme semble en apparence reconquérir son pouvoir d'agir, celui (ou celle) auquel se destine son jeu sexuel est libre de considérer cette sexualité comme auto-contrôlée ou hypersexualisée. Ce n'est pas la femme qui contrôle sa sexualité qui en juge. Le jugement reste évalué de l'extérieur, assujetti au regard et à l'interprétation d'autrui. En d'autres termes, même en tentant de changer l'image, on ne change pas celui qui la regarde. Tout est soumis à son pouvoir épistémique. Ainsi, la décolonisation de Séhérazade reste inexorablement inachevée. Le chapitre qui suit ne nous offre pas les clefs pour la poursuivre. L'appropriation de la sexualité de la femme arabe est pourtant importante pour mieux comprendre les limites de l'empowerment, car la mise en œuvre des pratiques visant à doter les femmes du tiers-monde d'une autonomisation procède du même processus de contrôle et reconduit des archétypes normatifs. 


\section{Care et empowerment}

Les chapitres V et VI critiquent le concept d'empowerment. Notion clef d'une stratégie de lutte à la pauvreté, il fait montre, en réalité, d'une entreprise sous-tendue par une colonialité $d u$ pouvoir, bien que son objectif soit d'instaurer un pouvoir d'agir de l'individu pour promouvoir son bien-être et le responsabiliser dans la lutte contre sa propre pauvreté. Mestiri démontre très finement comment cette stratégie traduit un rapport de force. La puissance d'agir étant un "pouvoir sur» (p. I06), l'empowerment est aussi pernicieux que contre-productif. Bien que la notion veuille redonner le pouvoir d'agir à des individus minorisés, dépolitisés, sa mise en œuvre tend, au contraire, à nier l'agentivité des femmes du tiers-monde en considérant l'existence de rapports sociaux de sexe patriarcaux qu'il faut déconstruire. En faisant comme si l'émancipation était réductible à une seule modalité, l'autrice nie leurs intérêts spécifiques et leur capacité à concevoir les modalités et les formes de leur pouvoir d'agir. L'empowerment traduit un nouvel ordre colonial et promotionne une vision de la vie bonne occidentale. Cette notion tend à être remplacée par le care.

Fondée sur la réciprocité et l'interdépendance entre les agents, l'approche du care paraît plus appropriée. Elle évite les pièges d'une approche surplombante fondée sur la charité envers les femmes du tiers-monde. Cependant, Mestiri considère que l'on ne s'écarte pas d'une autre forme de colonialité dans la mesure où le care s'opposerait à la justice. Ce faisant, elle reprend une discussion éculée et ce point de départ fausse le raisonnement et la conclusion qui en découle.

En effet si, lorsqu'elle a été formulée par Gilligan (I982), la notion de care était vue comme s'opposant à la justice libérale et critiquait les omissions du libéralisme politique, désormais la division entre le care et la justice ne s'applique plus. De nombreuses promotrices d'une politique du care sont revenues sur cette dichotomie pour la rejeter. En effet, care et justice participent conjointement à la détermination d'un ensemble de normes, et le care est une charpente importante dans la construction d'une société plus inclusive envers les personnes minorisées. Il ancre la justice sociale afin d'inciter à les prendre en compte. Le retour sur le rapport care - justice (et ce malgré la nuance apportée dans la note de bas de page $n^{\circ} 2$, p. I29), atteste de la lunette étriquée par laquelle Mestiri regarde le care. Elle s'explique par la référence à Narayan qui n'en est pas une s'agissant de la théorie du care.

En récusant le fait que le care serait plus à même d'outiller les femmes du tiers-monde, l'autrice procède d'une triple omission. En effet, contrairement à ce qui est suggéré, le care suppose des tiers-pourvoyeurs, comme en témoigne la notion de porte-voix de Kittay (I). Ensuite, elle est au cœur des valeurs des femmes du tiers-monde. Leur sororité repose sur l'autonomie relationnelle et soutient les mécanismes par lesquels elles résistent, survivent et s'autonomisent au quotidien. Ainsi, le care ne les sauve pas en étant imposé de l'extérieur puisqu'il est déjà inscrit au cœur de leurs rapports 
sociaux. Le care est une notion si profondément inscrite dans les valeurs et les modes de vie des femmes du tiers-monde que l'Occident ne peut venir le leur imposer (2). Enfin, lorsque Mestiri affirme que «la réponse [...] ne peut se situer que dans une politisation du care qui fait de l'empowerment l'incarnation de la réciprocité» (p. I36), elle néglige que la réciprocité est au cœur du care et en est un principe fondateur. Celle-ci n'est donc plus à repenser. Par conséquent, si le care peut, selon Narayan, présenter un discours colonial, il ne reconduit pas une colonialité du genre puisqu'il est une "disposition éthique liée au statut de dominé-e-s» (Dorlin, 2005, p. 88). Il ne cherche donc pas à les «encapaciter».

Selon les deux critiques qui viennent d'être formulées, on voit mal comment pourrait opérer le «féminisme de la frontière » dans la déconstruction de la colonialité $d u$ genre et de ce qu'elle suppose en termes de pouvoir et de savoir. Cependant, l'ouvrage présente l'avantage de nous faire réfléchir sur notre regard surplombant en ce qui concerne certaines femmes. Il nous oblige à dire où nous habitons le monde et par où nous le regardons. 